University of Nebraska - Lincoln

DigitalCommons@University of Nebraska - Lincoln

Robert Katz Publications

Research Papers in Physics and Astronomy

January 1989

Perspectives on the Development of Track Physics

Robert Katz

University of Nebraska-Lincoln, rkatz2@unl.edu

Follow this and additional works at: https://digitalcommons.unl.edu/physicskatz

Part of the Physics Commons

Katz, Robert, "Perspectives on the Development of Track Physics" (1989). Robert Katz Publications. 77. https://digitalcommons.unl.edu/physicskatz/77

This Article is brought to you for free and open access by the Research Papers in Physics and Astronomy at DigitalCommons@University of Nebraska - Lincoln. It has been accepted for inclusion in Robert Katz Publications by an authorized administrator of DigitalCommons@University of Nebraska - Lincoln. 
Published in Nuclear Tracks and Radiation Measurements 16:2/3 (1989), pp. 221-224; now known as Radiation Measurements; formerly International Journal of Radiation Applications and Instrumentation. Part D. Nuclear Tracks and Radiation Measurements.

Published by 1989 Pergamon Press plc. Used by permission.

http://www.sciencedirect.com/science/journal/13504487

Submitted November 28, 1988 .

\title{
Perspectives on the Development of Track Physics
}

\author{
Robert Katz \\ Department of Physics and Astronomy, University of Nebraska-Lincoln, \\ Lincoln, NE 68588-0111, U.S.A.
}

Let me thank all of you for being here, Tony Starace, for having conceived this meeting, and Bob Wood and Matesh Varma, for over 20 years of support without which there would have been no track physics. There are many others to thank: the students, postdoctorates, and senior visitors who actually did all the work, and the many investigators around the world who made measurements that proved to be essential to developing and testing the notions of particle tracks.

This enterprise began when I undertook to rewrite an introductory physics text by Henry Semat to adapt it to a calculus-based course. That became Physics, by Henry Semat and Robert Katz, published in 1958. In that writing I became persuaded of the beauty of the magnetic monopole as a pedagogic device in the teaching of elecricity and magnetism. Later I looked into special relativity to check the validity of my notions. That resulted in a Momentum book sponsored by the Commission of College Physics which was titled An Introduction to the Special Theory of Relativity. There I showed how easy it was to make relativistic transformations of the electric and magnetic fields, if only one admitted the use of poles. I may have been the first to write an explicit expression for the Lorentz force on a pole. That equation said that a moving pole would describe a helical path in a uniform electric field. This became one possible basis for the identification of the pole.

In the summer of 1958 I taught at the University of Illinois, and as luck would have it, Bob Hill-who had been my thesis advisor-was there. He was working with nuclear emulsions and became interested in my story about poles. Together we tried a hasty experiment exposing some nuclear emulsion (subject to an elecric field) to the Betatron, to look for a particle that moved in a circular path. The experiment was a failure, but then Bob Hill offered the notion that I should look into the track itself. That suggestion matured into track physics.

Sometime before, Dirac had shown quantum-mechanically that the existence of a quantized pole would justify a quantized electric charge. His required pole strength was large compared to the electron's charge. Consequently it would be heavily ionizing, since a moving magnetic pole generates an electric field just as a moving charge generates a magnetic field. Cosmic ray investigators had recently sent emulsions aloft in balloons and had observed very heavy tracks which they identified as being due to heavy nuclei. I wondered whether their identification was possibly in error, whether somewhere in these heavy tracks a pole was hiding undetected, because no one knew its signature.

I thought it would be an easy task to define the signature of a pole. I would look in the literature, find a theory of the tracks of heavy ions in emulsion, make a few changes and lo, the theory of the track of a pole. I found no such theory of heavy ion tracks. And so I set about making one.

At that time the physics department at Kansas State had no optical microscope. Through Bob Hill, I obtained some cosmic-ray-exposed emulsion, and ultimately the department bought a microscope. It was a long time before the model of a heavy ion track emerged. The essential ingredient was Jim Butts, who was my graduate student. He projected the image of tracks, via a mirror placed above a microscope eyepiece, onto pieces of paper, and traced around them to get data on the variation of track width with range. There were a couple of preliminary models around that had been applied to the stopping ends of tracks of lighter ions, neither one of which fitted these data. One model took as its criterion the electron flux, the other the energy flux, both from delta rays. Our change was to calculate the radial distribution of dose, and to see whether the track width could be associated with a fixed dose level. It worked. I remember my delight, speaking with Jim and Ed Kobetich, who was then an undergraduate, in which I allowed that we had struck gold. Then, I didn't know how right I was.

I had been teaching nuclear physics, and knew of roentgens, rads, and rems, and knew that I didn't understand those rems. I didn't understand RBE (relative 
biological effectiveness). I didn't understand quality factors. When we got our first good fit to heavy ion tracks, the light turned on. This had to be the basis of the rem. I told Jim Butts to go and find some biological data to test our track model. He turned up with some papers of Hutchinson and Pollard (1961a, b), on measurements of the cross section for the inactivation of dry enzymes and viruses. It was from Hutchinson's papers that I first learned of the target theory. We applied our model of tracks in emulsion, and within a very short time-perhaps a month or two-Jim had a model of these cross sections that fitted the data. Our constraint then, as now, was that the response to delta rays as a function of radial dose had to be the same as the response to gamma rays. The reasoning was simple. In both cases the electrons did the inactivating. And the enzyme could not know the difference in the sources of the electrons. We had a theory of RBE for the inactivation of dry enzymes and viruses (Butts and Katz, 1967).

In so doing we turned the model of Lea (1962), which preceded us, upside down. His notion, and that of Hutchinson, was that the cross section should be principally determined by the size of the molecule, with some correction for delta rays, which he called the "associated volume." The experimenters had sought to measure virus size by a radiation experiment, expecting that the cross section would saturate with an increase in projectile LET at the cross sectional area corresponding to the associated volume. But there was no saturation. The cross section kept increasing.

Later, when virus sizes were known from other means, these inactivation cross sections proved to be orders of magnitude greater than the virus size. The basic difference between the ideas of Lea and mine arose from the fact that he came to the problem from an observation of thin tracks and I came to it from the observation of fat tracks, in a medium in which the targets were small: the heavy primary cosmic ray tracks in nuclear emulsions.

We had turned Lea's idea upside down. We assumed the target to be a point, and attributed the cross section entirely to the effect of delta rays. And the problems that people had with understanding these data disappeared. We had invented the track theory of a 1-hit detector. Later these ideas were applied to other detectors, like scintillation counters, and to an improved theory of particle tracks in nuclear emulsions by Ed Kobetich and myself (Katz and Kobetich, 1969), Ed now being a graduate student at Nebraska. Our new theory of tracks in emulsions incorporated ideas we had developed in the theory of RBE for dry enzymes and viruses, and now included the tracks of lightly ionizing particles like protons, as well as of heavily ionizing particles. It allowed extension to the concept that a desensitized emulsion was a many-hit detector, and later to a visualization of what the track of a heavy particle in an "emulsion" of biological cells might look like.
I learned of a succession of meetings in interesting places: Radiation Research in Cortina d'Ampezzo, an L. H. Gray meeting on Tracks in Cambridge, England, and a Radiation Research Society meeting in Puerto Rico. I resolved to go to these interesting places with my message. Typically, the initial response seemed to me to be disinterest. But I persisted. In a search for funding, I sent letters to a large number of agencies asking for support on the basis of the enzyme-and-virus paper. Only one project director picked it up, very much to his credit. Nat Barr at DOE wrote back suggesting that I write a small proposal. I later found out that people returning from Cortina d'Ampezzo had been talking about my paper there-not necessarily agreeing, but at least talking about it. That was the beginning of my marriage to DOE that has now lasted over 20 years, with the help of Bob Wood and Matesh Varma.

The way in which this work proceeded was that whatever I undertook always seemed to be the wrong thing according to my critics. When we had done the model for dry enzymes and viruses in the point target approximation, I was told that we should have taken target size into account. When we had developed an extended target model, I was told that there was little interest in enzymes. Cells were the important thing. That was how I learned of the existence of data for cellular survival after irradiation with beams of heavy ions. Here the principal stimulus to further work was the work of Larry Powers and others (1968) on bacterial spores. I studied these data and could not understand them at all. Finally came dawn. At low LET we were seeing the grain count regime, with inactivated spores like beads on a string. And the string became fuller and fuller with an increase in LET. Finally, with very heavy ions, we entered the track width regime. With beams of ions there could be an accumulation of damage from the delta rays from different ions. Delta rays from a second ion could inactivate spores which survived the damage from a first ion, though storing it. In this case it was the accumulation of damage from the delta rays of different ions that produced the end result. We named these processes "gamma-kill" and "ion-kill," the first rather like the effect of overlapping electron tracks in a gamma ray irradiation, while the second represented the damage done by single ions. The concept resulted in a model with four parameters (Katz et al., 1971), through which we fitted the data rather well. In emulsions, only three parameters were required, one of which represented the grain size. For cells, I imagined that the sensitive volume within the nucleus was rather like beans in a bean bag. Two size parameters were required-one for the beans, the other for the bean bag. Then I heard endless remarks about four parameters, and how with that many, one could fit an elephant. In the many years that have since elapsed, no model has arisen that fit the data as well, whether with four or fewer parameters. Newer models seem to have 
many more parameters than mine. And as yet I have not seen as extensive an attempt to fit the experimental data.

When we had developed a model for cellular inactivation by heavy ions, I was told that the real issue was cell transformation, and when we made some inroads into the application of our model to transformations, I was told that we really should try to get at cancer induction and that the other things were of much lesser importance. We have tried to do something with such data, but the available data have been of too limited dynamic range and have not been internally consistent. I hope that one day, when new data are available, we will have an opportunity to try to fit them.

Our work has been criticized because we deal in parameters. We have constructed a parametric model for enzymes and viruses, for scintillators, for emulsions, for TLDs, for heavy ion radiolysis, and for a host of other phenomena. We have been criticized for using what has been called an amorphous track model, in which we calculated the average radial dose distribution to calculate activation cross sections. We have been criticized for using gamma rays as a calibrating radiation. Nevertheless our models and calculations have agreed with much (though not all) of the data. We have been told that models that agree with data are not necessarily right. To which I have replied that all that can be said for models that agree with data, is that they are likely to compete favorably with models that do not agree with data.

Other investigators are persuaded that stochastics are central to radiation effects, and that averages can tell us nothing. I do not agree. There are phenomena for which averages are a proper description. There are other phenomena for which one must focus on the fluctuations. In exposing a photographic film we measure the average light intensity. But when we are concerned with graininess we must know the fluctuation in photon flux incident on the film. But this is a higher order effect. There is some question as to whether we yet have proper first order models of track effects, especially in radiobiology. I do not think the data yet have sufficient precision for the study of second order effects. But still we must be aware that there are important phenomena which are wholly dependent on fluctuations. Thermal noise is one. I think black body radiation is another.

Models need a basis for calibration. Thus far, the only reliable calibrating information about a detector is its response to gamma rays. We have no data from which to make the transition from energy deposition in small volumes (presumed to represent an unidentified small target) to the observed effect. Models of radiation effects should parallel physical experiments, in which the central result is the determination of a cross section. Models or experiments which do not yield a cross section seem to me to be inadequate. Response measured as a function of dose seems adequate for irradiation with $\mathrm{X}$ - and gamma rays, or with energetic electron beams, where the electron slowing-down spectra are comparable, but this seems to be a very misleading basis for comparison with heavy ions or neutrons.

Other investigators favor a formula which they call the alpha-beta model, where the negative logarithm of the surviving fraction of cells as a function of dose is given as $\alpha D+\beta D^{2}$. This is really nothing more than the first two terms of a Taylor expansion, and is adequate to fit data of limited dynamic range, but typically fails when the dynamic range is extended. But this is not a model. It is rather like a set of French curves. There has not yet emerged a model from which we can calculate both alpha and beta, for radiations of different quality, which agrees with such data. It is a convenient spline fit. Its persuasiveness is frequently based on its fit to survival data at low dose. But this is where the experiments are weakest. Witness the emergence of radiation hormesis as a low dose phenomenon, but one which is difficult to support logically. There are times when statistical fitting criteria are very misleading. My test of the validity of statistical criteria is to find a perfectly absurd proposition and ask whether its experimental results satisfy statistical criteria. I regard the success of ESP, which to me is nonsense, as a test of the validity of statistical criteria rather than a test of the existence of the phenomenon.

One must understand that in science it is necessary to formulate models that are falsifiable. In radiation research there are many qualitative arguments based on such words as "might be" and "could be," but few quantitative and falsifiable models. We should keep in mind that the mechanistic models most people desire have not emerged in the 20 years I have been working. But parametric models have made enormous progress. In favor of parametric models, I must add that the data of radiation effects are typically bland, typically structure-free, and typically of limited dynamic range. If one is to test a model, it must be tested on a wide range of detectors having widely different properties, and this can only be done with a global parametric model. Keep in mind that mechanistic models cannot be global, nor can parametric models be mechanistic.

One must keep in mind also that this is the route physics has travelled in the past. One sorts out data via parametric formulae before mechanistic models can be created. If there had been no Balmer, there would have been no Bohr. If there had not been a Bohr there would not have been a Schrödinger. Today, many physicists believe that all must proceed from first principles, neatly derived from quantum mechanics. In so complex a subject as radiobiology, this is impossible. We are lucky to have a global parametric model. Perhaps the success of parametric models will stimulate the development of mechanistic ideas in the many detectors to which track physics has been applied. Perhaps it will serve to emphasize that models must be relevant before we are overly concerned with their precision. 
This has been almost 25 years of great sport. The models my students, my collaborators, and I have developed are phenomenological and parametric. They could not have been developed without an abundance of data. I have been lucky that in my time heavy ion accelerators have become available, and from them much data on the response of detectors to energetic heavy ions has been acquired. With each new, relevant bit of published research, I have sought to examine whether track theory was applicable. We have found 1-hit detectors, 2hit detectors, and even up to 6- or 8-hit detectors, whose response has been reasonably well described by the theory. None of this could have been done without the enormous amount of solid experimental work of others. None of it would have been done without the efforts of other model-builders whose work and whose criticism stimulated my own. To all of you, those here and those absent, my best thanks. None of this could have been done without you.

\section{References}

Butts J. J. and Katz R. (1967) Theory of RBE for heavy ion bombardment of dry enzymes and viruses. Radiat. Res. 30, 855-871.

Hutchinson F. and Pollard E. (196la) Physical principles of radiation action. In: Mechanisms in Radiobiology (Edited by Errara M. and Forssberg A.), Vol. 1, pp. 1-66. Academic Press, New York.

Hutchinson F. and Pollard E. (1961b) Target theory and radiation effects on biological molecules. In: Mechanisms in Radiobiology (Edited by Errara M. and Forssberg A.), Vol. 1, pp. 71-91. Academic Press, New York.

Katz R. and Kobetich E. J. (1969) Particle tracks in emulsion. Phys. Rev. 186, 344-351.

Katz R., Ackerson B., Homayoonfar M., and Sharma S. C. (1971) Inactivation of cells by heavy ion bombardment. Radiat. Res. 47, 402-05.

Lea D. E. (1962) Actions of Radiations on Living Cells. Cambridge University Press, U.K.

Powers E. L., Lyman J. T., and Tobias C. A. (1968) Some effects of accelerated charged particles on bacterial spores. Int. J. Radiat. Biol. 14, 313-330. 